\title{
LA EMIGRACIÓN ESPAÑOLA ASISTIDA A LATINOAMÉRICA, 1968-1990
}

\author{
Salvador Palazón Ferrando
}

\section{RESUMEN}

Este artículo trata de resaltar la evolución decreciente que ha seguido la corriente emigratoria española asistida a Latinoamérica en los últimos veinte años, a causa de la crisis socio-económica que sufre este área; asimismo, analiza el perfil socio-demográfico del emigrante español, los países de destino preferente y las regiones españolas que más han contribuido a alimentar esta corriente.

\section{SUMMARY}

This paper support the decreasing evolution of the spanish emigration trend to Latin America in the last twentieth years, be cause of the social and economic crisis in this area. Another aims is the analysis of the social and demographic characteristics of spanish emigrants, the countries that have received and the spanish regions that have expelled the main number of emigrants.

\section{Fuentes}

Para el estudio de la emigración española asistida ${ }^{1}$ en este período, debemos acudir a los datos que sobre la misma publica el Instituto Español de Emigración (I.E.E.) en sus publicaciones:

- Emigración española asistida, años 1968-1980. (E.E.A.).

- Datos estadísticos sobre la emigración española, años 1981-1986. (D.E.E.E.).

- Memoria anual del Instituto Español de Emigración, años 1987-1988. (M.A.I.E.E.).

- Datos estadísticos sobre la emigración española, año 1989. (D.E.E.E.).

- Anuario de Migraciones, 1992². (A.M.).

1 Por «asistido» se entiende a todo emigrante que solicitaba y/o recibía ayuda del Instituto Español de Emigración para realizar su desplazamiento.

2 Este anuario aparece publicado por la Dirección General de Migraciones (D.G.M.), nueva denominación del I.E.E. 
Estas estadísticas del I.E.E. presentan los siguientes inconvenientes para el análisis de la emigración española a Latinoamérica, inconvenientes que determinan la ruptura con la serie estadística iniciada en 1946 sobre el movimiento migratorio español a Latinoamérica y que impiden cualquier tipo de comparación entre este período y el anterior a 1968, pues:

$1^{\circ}$ ) Los emigrantes asistidos representan de 1968 a 1971 entre un $40 \%$ y un $50 \%$ del total de emigrantes españoles a Latinoamérica, y a partir de 1971 será el único tipo de emigrantes reconocido por la nueva Ley de Emigración española ${ }^{3}$. Esta nueva concepción de la emigración española supone una pérdida de información, así como una alteración de los resultados en todos los niveles: volumen de emigrantes, países de destino, grupos profesionales y regiones de procedencia (PALAZÓN, 1989a, 177-184).

$2^{\circ}$ ) No se ofrecen datos sobre el retorno, o los que se ofrecen no son fiables porque están asentados en las bajas consulares ${ }^{4}$.

$3^{\circ}$ ) No se ofrecen datos para tres países: Honduras, Nicaragua y Paraguay, aunque las bajísimas cifras de emigrantes que se supone se dirigen a estos países no deben alterar demasiado los resultados finales.

$4^{\circ}$ ) No existe una serie continua sobre la edad y sexo de los emigrantes a Latinoamérica, lo que impide analizar estas dos características.

$5^{\circ}$ ) La distribución por categorías profesionales sólo abarca hasta 1988, y para algunos países no se dispone de datos para los últimos años ${ }^{5}$.

\section{Evolución de la corriente emigratoria española asistida, 1968-1990}

La emigración española asistida a Latinoamérica entre 1968 y 1990 ha experimentado un descenso continuado de sus efectivos, hasta el punto que estas cifras se sitúan por debajo de los mil emigrantes anuales a partir de 1983 (Cuadro I). Este descenso se explica por el empeoramiento de la situación socio-económica y política en la mayoría de los países latinoamericanos, junto con la política inmigratoria selectiva llevada a cabo por los mismos.

Tres hechos corroboran que sea el empeoramiento de la situación socio-económica en Latinoamérica la causa del descenso de la emigración española hacia ese área:

$1^{\circ}$ ) En España no se ha logrado el pleno empleo, por el contrario la cifra de parados ha aumentado hasta superar los tres millones.

$2^{\circ}$ ) El fracaso de los intentos por aumentar la inmigración europea realizados por Argentina y Venezuela, países tradicionalmente receptores de la emigración española ${ }^{6}$.

3 Hasta 1971 legalmente se contabilizaban dos tipos de emigrantes: los espontáneos y los asistidos.

4 La escasa fiabilidad de las bajas consulares para determinar el retorno viene establecida porque no todos los españoles se dan de alta en los consulados españoles al emigrar a América Latina, y porque en las bajas consulares también se incluyen los fallecidos.

5 Tal es el caso de El Salvador entre 1985 y 1989, y la República Dominicana en 1988.

6 Varios son los ejemplos que en este sentido presentan ambos países. En 1970, Argentina entre las directrices de su política nacional expone la necesidad de «impulsar la inmigración de carácter selectivo, procurando mantener la actual composición étnica de la población, y teniendo en cuenta los requerimientos del desarrollo y la seguridad»; en 1973, el Plan Trienal para la Reconstrucción y Liberación Nacional del Gobierno argentino propone como uno de los objetivos demográficos aumentar y orientar la inmigración (I.E.E., 1985, 51- 
$3^{\circ}$ ) La inversión de la corriente migratoria, al ser ahora los latinoamericanos quienes emigran hacia España; de hecho, la población latinoamericana residente en España se ha cuadruplicado en las dos últimas décadas al pasar de 42.260 en 1970 (D.G.M., 1991, 103) a 220.000 en $1990^{7}$.

CuAdro I

EVOLUCIÓN ANUAL DE LA EMIGRACIÓN ESPAÑOLA ASISTIDA A LATINOAMÉRICA (1968-1990)

\begin{tabular}{|lrrr|}
\hline AÑ & TOTAL & AÑ & TOTAL \\
\hline 1968 & 8.423 & 1981 & 1.499 \\
1969 & 8.257 & 1982 & 1.255 \\
1970 & 5.314 & 1983 & 998 \\
1971 & 4.855 & 1984 & 887 \\
1972 & 4.067 & 1985 & 690 \\
1973 & 2.708 & 1986 & 694 \\
1974 & 2.429 & 1987 & 690 \\
1975 & 2.604 & 1988 & 764 \\
1976 & 2.494 & 1989 & 574 \\
1977 & 2.461 & 1990 & 322 \\
1978 & 1.982 & & \\
1979 & 1.820 & & \\
1980 & 1.051 & & \\
\hline
\end{tabular}

FuENTE: E.E.A., 1968-1980; D.E.E.E., 1980-1986 y 1989; M.A.I.E.E., 1987-1988; A.M., 1992. Elaboración propia.

Este descenso de los aportes emigratorios con destino a América Latina ha tenido como consecuencia, junto con el envejecimiento de la colonia española y el retorno, un importante descenso de la presencia española en América Latina, descenso que afecta de manera más notoria a los países con mayor volumen de españoles asentados, sobre todo, en aquellos donde el contingente llegado con anterioridad a 1935 constituye una parte considerable del total (es el caso de Argentina, Brasil, Cuba, Uruguay y Chile) ${ }^{8}$ (Cuadro II) (PALAZÓN, 1989b).

52); y en 1977, un nuevo Decreto de 29 de diciembre favorecerá la llegada e instalación de inmigrantes que deseasen dedicarse al desarrollo de diversas áreas económicas de interés para el país (industria, agricultura, minería, pesca, etc.). Algo similar ocurre en Venezuela, que en 1979 firma con España un «Convenio sobre Migración» destinado a facilitar la contratación de trabajadores cualificados mediante reclutamiento, selección, traslado y colocación de los mismos, así como la formación profesional de la fuerza de trabajo venezolana mediante el aporte inmigratorio español (B.O.E., 16-II- 1981).

7 Las cifras oficiales españolas dan para el año 1990 un total de 59.439 inmigrantes latinoamericanos (D.G.M., 1991, 103), pero como éstos son únicamente los que tienen su situación legalizada debemos sumar como mínimo a cada uno de ellos otros 2,7 inmigrantes ilegales, tal y como indica Cáritas Española en un estudio publicado en 1987 (GOZÁLVEZ PÉREZ, 1990, 18-19). Para más datos sobre la inmigración extranjera en España véase GOZÁLVEZ PÉREZ (1990) y GOZÁLVEZ PÉREZ (1991).

8 Venezuela no entra en este conjunto porque el grueso de la población española asentada en él llega con posterioridad a 1950 y todavía no se ha visto afectada por problemas de envejecimiento que condicionen su pervivencia. 
Figura 1. Evolución anual de la emigración española asistida a Latinoamérica, 1968-1990.

CuAdro II

POBLACIÓN ESPAÑOLA RESIDENTE EN AMÉRICA LATINA (1960-1980)

\begin{tabular}{|lccc|}
\hline PAís & $\begin{array}{c}\text { AÑ } \mathbf{1 9 6 0} \\
\text { POBLACIÓN } \\
\text { ESPAÑOLA }\end{array}$ & $\begin{array}{c}\text { AÑO 1970 } \\
\text { POBLACIÓN } \\
\text { ESPAÑOLA }\end{array}$ & $\begin{array}{c}\text { AÑO 1980 } \\
\text { POBLACIÓN } \\
\text { ESPAÑOLA }\end{array}$ \\
\hline ARGENTINA & 715.685 & 540.100 & 363.550 \\
BOLIVIA & $1.000^{*}$ & $1.200^{*}$ & 1.394 \\
BRASIL & 144.080 & 115.893 & 98.515 \\
COLOMBIA & $6.000^{*}$ & $3.000^{*}$ & $3.000^{*}$ \\
COSTA RICA & $1.000^{*}$ & 1.335 & 1.546 \\
CUBA & $74.000^{*}$ & 74.026 & $60.000^{*}$ \\
CHILE & 21.777 & 16.628 & 12.290 \\
ECUADOR & $750^{*}$ & $2.000^{*}$ & 2.591 \\
GUATEMALA & $1.000^{*}$ & 1.700 & 1.405 \\
MÉXICO & 49.637 & 31.038 & 31.363 \\
NICARAGUA & $250^{*}$ & 546 & $475^{*}$ \\
PANAMÁ & 2.292 & 2.793 & 2.706 \\
PARAGUAY & $1.000^{*}$ & 1.057 & 1.160 \\
PERÚ & $5.500^{*}$ & 5.446 & 4.723 \\
R. DOMINICANA & 4.060 & 2.318 & $2.000^{*}$ \\
EL SALVADOR & $500^{*}$ & $750^{*}$ & $750^{*}$ \\
URUGUAY & $72.754^{*}$ & 44.831 & 31.546 \\
VENEZUELA & 166.660 & 149.747 & 141.760 \\
TOTAL APROX. & 1.260 .000 & 1.000 .000 & 800.000 \\
\hline
\end{tabular}

* Estimaciones. Sin datos para Honduras, Puerto Rico.

Fuente: Censos Nacionales de Población. Elaboración propia. 
Figura 2. Distribución de los españoles de nacimiento censados en América Latina hacia los años 1960 y 1980, según el país de residencia.

\section{Países de destino de la emigración española asistida}

En conjunto, el principal país de destino de la emigración española asistida en este período es Venezuela, que recibe casi a la mitad del total de emigrantes del período. Argentina pasa a ser un destino secundario de la emigración española, y a ella tan sólo se dirigen un $12 \%$ de los emigrantes. De los demás países únicamente cabe destacar a Brasil, México y Perú que absorben entre un 5\% y $10 \%$ de la emigración española a Latinoamérica (Cuadro III).

Esta distribución difiere de una a otra etapa; así, en 1968- 75 y 1976-80, Venezuela es el primer destino de la emigración española, pues, debido sobre todo a la reagrupación familiar, acoge a más de la mitad de los emigrantes españoles que se dirigen a Latinoamérica; sin embargo, a partir de 1981, la crisis económica que afecta a Venezuela ${ }^{9}$ tendrá como consecuencia su pérdida de atractivo para el emigrante español y el fuerte descenso de su participación entre los destinos latinoamericanos de la emigración española. Al mismo tiempo, la reducción generalizada del número de emigrantes provocará que destinos tradicionalmente periféricos en la corriente emigratoria española a Latinoamérica adquie-

9 La caída de los precios del petróleo en el mercado internacional y la reducción de la cuota de producción asignada al país por la OPEP generaron, a partir de 1981, una grave crisis económica que se tradujo en: $1^{\circ}$ ) una reducción del gasto público, paralizándose proyectos en realización o aplazándose otros previstos; $2^{\circ}$ ) un aumento del endeudamiento externo, que compromete la capacidad inversora y de gasto del Estado; $3^{\circ}$ ) una contracción del importante sector de la construcción; $4^{\circ}$ ) una inestabilidad del sector inversor y financiero privado; $5^{\circ}$ ) un incremento del desempleo y la subocupación. (TORREALBA, 1986, 121-122). 
Cuadro III

PAÍSES DE DESTINO DE LA EMIGRACIÓN ESPAÑOLA A LATINOAMÉRICA (1968-1990)

(Cifras absolutas y relativas)

\begin{tabular}{|lrrrrr|}
\hline PAÍS & $\mathbf{1 9 6 8 - 7 5}$ & $\mathbf{1 9 7 6 - 8 0}$ & $\mathbf{1 9 8 1 - 8 5}$ & $\mathbf{1 9 8 6 - 9 0}$ & $\mathbf{1 9 6 8 - 9 0}$ \\
\hline ARGENTINA & 5.411 & 542 & 589 & 224 & 6.766 \\
BOLIVIA & 391 & 220 & 243 & 135 & 989 \\
BRASIL & 3.397 & 523 & 275 & 135 & 4.330 \\
COLOMBIA & 1.287 & 328 & 568 & 239 & 2.422 \\
COSTA RICA & 150 & 44 & 167 & 51 & 412 \\
CUBA & 8 & 18 & 27 & 59 & 112 \\
CHILE & 852 & 244 & 198 & 169 & 1.463 \\
ECUADOR & 488 & 324 & 535 & 577 & 1.924 \\
GUATEMALA & 355 & 93 & 147 & 97 & 692 \\
MÉXICO & 3.565 & 944 & 264 & 392 & 5.165 \\
PANAMÁ & 561 & 87 & 73 & 53 & 774 \\
PERÚ & 1.127 & 551 & 790 & 396 & 2.864 \\
PUERTO RICO & 163 & 38 & 31 & 81 & 313 \\
R. DOMINICANA & 477 & 157 & 208 & 181 & 1.023 \\
EL SALVADOR & 114 & 106 & 12 & 2 & 234 \\
URUGUAY & 662 & 72 & 115 & 50 & 899 \\
VENEZUELA & 19.649 & 5.517 & 1.087 & 203 & 26.456 \\
\hline TOTAL & 38.657 & 9.808 & 5.329 & 3.044 & 56.838 \\
\hline ARGENTINA & 14,0 & 5,5 & 11,1 & 7,4 & 11,9 \\
BOLIVIA & 1,0 & 2,2 & 4,6 & 4,4 & 1,7 \\
BRASIL & 8,8 & 5,3 & 5,2 & 4,4 & 7,6 \\
COLOMBIA & 3,3 & 3,3 & 10,7 & 7,9 & 4,3 \\
COSTA RICA & 0,4 & 0,4 & 3,1 & 1,7 & 0,7 \\
CUBA & 0,0 & 0,2 & 0,5 & 1,9 & 0,2 \\
CHILE & 2,2 & 2,5 & 3,7 & 5,6 & 2,6 \\
ECUADOR & 1,3 & 3,3 & 10,0 & 19,0 & 3,4 \\
GUATEMALA & 0,9 & 0,9 & 2,8 & 3,2 & 1,2 \\
MÉXICO & 9,2 & 9,6 & 5,0 & 12,9 & 9,1 \\
PANAMÁ & 1,5 & 0,9 & 1,4 & 1,7 & 1,4 \\
PERÚ & 2,9 & 5,6 & 14,8 & 13,0 & 5,0 \\
PUERTO RICO & 0,4 & 0,4 & 0,6 & 2,7 & 0,6 \\
R. DOMINICANA & 1,2 & 1,6 & 3,9 & 5,9 & 1,8 \\
EL SALVADOR & 0,3 & 1,1 & 0,2 & 0,1 & 0,4 \\
URUGUAY & 1,7 & 0,7 & 2,2 & 1,6 & 1,6 \\
VENEZUELA & 50,8 & 56,3 & 20,4 & 6,7 & 46,5 \\
\hline TOTAL & 100,0 & 100,0 & 100,0 & 100,0 & 100,0 \\
\hline
\end{tabular}

FuENTE: E.E.A., 1968-1980; D.E.E.E., 1980-1986 y 1989; M.A.I.E.E., 1987-1988; A.M., 1992. Elaboración propia. 
FIGURA 3. Países de destino de la emigración española asistida a Latinoamérica, 1968-1990.

CuAdro IV

EMIGRACIÓN ESPAÑOLA ASISTIDA A LATINOAMÉRICA SEGÚN ETAPA DE LLEGADA (1968-1990) (Cifras relativas)

\begin{tabular}{|c|c|c|c|c|c|}
\hline PAÍS & 1968-75 & 1976-80 & 1981-85 & 1986-90 & TOTAL \\
\hline ARGENTINA & 80,0 & 8,0 & 8,7 & 3,3 & 100,0 \\
\hline BOLIVIA & 39,5 & 22,2 & 24,6 & 13,7 & 100,0 \\
\hline BRASIL & 78,5 & 12,1 & 6,4 & 3,1 & 100,0 \\
\hline COLOMBIA & 53,1 & 13,5 & 23,5 & 9,9 & 100,0 \\
\hline COSTA RICA & 36,4 & 10,7 & 40,5 & 12,4 & 100,0 \\
\hline CUBA & 7,1 & 16,1 & 24,1 & 52,7 & 100,0 \\
\hline CHILE & 58,2 & 16,7 & 13,5 & 11,6 & 100,0 \\
\hline ECUADOR & 25,4 & 16,8 & 27,8 & 30,0 & 100,0 \\
\hline GUATEMALA & 51,3 & 13,4 & 21,2 & 14,0 & 100,0 \\
\hline MÉXICO & 69,0 & 18,3 & 5,1 & 7,6 & 100,0 \\
\hline PANAMÁ & 72,5 & 11,2 & 9,4 & 6,8 & 100,0 \\
\hline PERÚ & 39,4 & 19,2 & 27,6 & 13,8 & 100,0 \\
\hline PUERTO RICO & 52,1 & 12,1 & 9,9 & 25,9 & 100,0 \\
\hline R. DOMINICANA & 46,6 & 15,3 & 20,3 & 17,7 & 100,0 \\
\hline EL SALVADOR & 48,7 & 45,3 & 5,1 & 0,9 & 100,0 \\
\hline URUGUAY & 73,6 & 8,0 & 12,8 & 5,6 & 100,0 \\
\hline VENEZUELA & 74,3 & 20,9 & 4,1 & 0,8 & 100,0 \\
\hline TOTAL & 68,0 & 17,3 & 9,4 & 5,4 & 100,0 \\
\hline
\end{tabular}

FuENTE: E.E.A., 1968-1980; D.E.E.E., 1980-1986 y 1989; M.A.I.E.E., 1987-1988; A.M., 1992. Elaboración propia.

ran cierta relevancia a partir de 1981, caso de Colombia, Ecuador o Perú (Cuadro III).

Otra de las consecuencias de la crisis económica que abate América Latina en las últimas décadas y del empeoramiento consiguiente de la situación socio-económica, ha sido la concentración de la mayor parte de la emigración española a Latinoamérica en la 
etapa anterior a 1975; de hecho, más de las dos terceras partes de los emigrantes llegan en esta etapa, un 17\% entre 1976-80, otro 9\% entre 1981-85, y el restante 5\% entre 1986-90 (Cuadro IV).

Por países, a excepción de Costa Rica, Cuba y Ecuador, la mayor parte de la emigración española llega en la primera etapa, 1968-75. En cuatro de estos países (Colombia, Chile, Guatemala y Puerto Rico) la proporción del contingente emigratorio que llega entre 1968-75 supera la mitad del total del período, e incluso en otros seis (Argentina, Brasil, México, Panamá, Uruguay y Venezuela), entre los que se encuentran los que habían sido anteriormente destinos preferentes de la emigración española, esta relación está por encima de los dos tercios (Cuadro IV).

\section{Tasas de actividad y distribución profesional de la emigración española asistida}

En general, algo más de la mitad de los emigrantes españoles asistidos que se dirigen en este período a Latinoamérica es población activa, proporción que no es mayor debido a la elevada participación de mujeres sin profesión y niños menores de quince años, es decir aquellos que participan del proceso de reagrupación familiar (Cuadro V).

Ahora bien, por etapas puede comprobarse como el descenso del reagrupamiento familiar ante situaciones cada vez menos favorables para el asentamiento definitivo del emigrante español, determina una subida de las tasas de actividad por encima del $70 \%$ a partir de 1981 (Cuadro V).

Por países, la tasa de actividad de la emigración española asistida supera el $50 \%$ en todos, a excepción de Venezuela y México, donde el fenómeno de la reagrupación familiar, sobre todo en el primero de ellos, adquiere mayores dimensiones y da lugar a tasas de actividad más bajas que en los demás casos (39\% y 49\%, respectivamente). Por otra parte, algunos países presentan tasas de actividad muy elevadas, caso de Bolivia, Cuba, Chile, Ecuador, Guatemala, Perú, Puerto Rico y la República Dominicana, donde como mínimo 7 de cada diez españoles declara alguna profesión al emigrar (Cuadro VI).

La demanda de mano de obra inmigrante cualificada llevada a cabo por los países latinoamericanos, así como la internacionalización de sus economías, ha determinado que los dos grupos profesionales que más emigrantes presentan sean el de profesionales y técnicos, por un lado, y, el de artesanos y obreros industriales, por otro. Sin embargo, la crisis económica que sufren estos países ha generado, en las etapas posteriores a 1981, un aumento de la proporción de profesionales y técnicos, que pasan a representar más del $70 \%$ de la emigración activa, en detrimento de los demás grupos profesionales (Cuadro V).

El grupo de profesionales y técnicos está compuesto, principalmente, por el personal cualificado que desplazan a Latinoamérica empresas españolas que desarrollan sus actividades en el campo de las obras públicas, de las instalaciones industriales o mecánicas ${ }^{10}$, y de las finanzas ${ }^{11}$, aunque también se incluyen en el mismo:

a) El personal cualificado que se desplaza para trabajar en empresas de capital latinoamericano o mixto.

b) Los miembros del clero con titulación universitaria que se dirigen a Latinoamérica para realizar sus tareas de apostolado y enseñanza.

10 Entre esas empresas encontramos a: Dragados y Construcciones, Entrecanales, Agromán, Huarte, Sereland, Artavol, Cubiertas y MZOV, Prensados y Aceros, Crespo y Blasco, Saetrame, Asland, Reinhold, Auxini, Sadespa, etc.

11 Es el caso del Banesto, Banco Central, Banco Exterior de España, Banco Popular, Banco de Santander, Banco Hispanoamericano, etc. 


\section{CUADRO V}

TASAS DE ACTIVIDAD Y DISTRIBUCIÓN POR RAMAS DE LA EMIGRACIÓN ESPAÑOLA A LATINOAMÉRICA SEGÚN PERÍODO (1968-1988)

(Cifras absolutas y relativas)

\begin{tabular}{|crrrrr|}
\hline RAMAS & $\mathbf{1 9 6 8 - 7 5}$ & $\mathbf{1 9 7 6 - 8 0}$ & $\mathbf{1 9 8 1 - 8 5}$ & $\mathbf{1 9 8 6 - 8 8}$ & TOTAL \\
\hline 1 & 6.284 & 2.494 & 2.771 & 1.262 & 12.811 \\
2 & 88 & 4 & 5 & 0 & 97 \\
3 & 1.384 & 366 & 212 & 59 & 2.021 \\
4 & 1.437 & 230 & 58 & 11 & 1.736 \\
5 & 2.930 & 399 & 28 & 7 & 3.364 \\
6 & 73 & 40 & 57 & 6 & 176 \\
7 & 394 & 55 & 0 & 2 & 451 \\
8 & 3.876 & 1.097 & 605 & 281 & 5.859 \\
9 & 420 & 80 & 11 & 5 & 516 \\
X & 1.770 & 384 & 151 & 42 & 2.347 \\
\hline \multirow{2}{*}{ TASA ACTIVIDAD } & 48,3 & 52,5 & 73,1 & 78,0 & 52,5 \\
\hline \multirow{2}{*}{ RAMAS } & $\mathbf{1 9 6 8 - 7 5}$ & $\mathbf{1 9 7 6 - 8 0}$ & $\mathbf{1 9 8 1 - 8 5}$ & $\mathbf{1 9 8 6 - 8 8}$ & TOTAL \\
\hline & & & & & \\
1 & 33,7 & 48,4 & 71,1 & 75,3 & 43,6 \\
2 & 0,5 & 0,1 & 0,1 & 0,0 & 0,3 \\
3 & 7,4 & 7,1 & 5,4 & 3,5 & 6,9 \\
4 & 7,7 & 4,5 & 1,5 & 0,7 & 5,9 \\
5 & 15,7 & 7,7 & 0,7 & 0,4 & 11,5 \\
6 & 0,4 & 0,8 & 1,5 & 0,4 & 0,6 \\
7 & 2,1 & 1,1 & 0,0 & 0,1 & 1,5 \\
8 & 20,8 & 21,3 & 15,5 & 16,8 & 19,9 \\
9 & 2,3 & 1,6 & 0,3 & 0,3 & 1,8 \\
X & 9,5 & 7,5 & 3,9 & 2,5 & 8,0 \\
\hline \multirow{2}{*}{ TOTAL } & 100,0 & 100,0 & 100,0 & 100,0 & 100,0 \\
\hline
\end{tabular}

1. Profesionales y Técnicos; 2. Administradores, gerentes y directores; 3. Empleados de Oficina; 4. Vendedores; 5. Agricultores, pescadores, cazadores, y silvicultores; 6. Minas y Canteras; 7. Transporte y comunicaciones; 8. Artesanos y obreros industriales; 9. Otros Servicios, deportes y diversiones; X. No clasificables.

FuENTE: E.E.A., 1968-1980; D.E.E.E., 1980-1986 y 1989; M.A.I.E.E., 1987-1988; A.M., 1992. Elaboración propia.

c) Personal sanitario (médicos, ATS, etc.).

d) Los Becarios del «Servicio de Emigración Cualificada y Cooperación Social» del I.E.E., que se trasladaban para impartir docencia universitaria o para dar asistencia profesional auxiliar.

Por países, el reparto de los emigrantes españoles en los distintos grupos profesionales difiere de unos casos a otros; así, el grupo de profesionales y técnicos representa más de las tres cuartas partes de la emigración española activa en Bolivia, Colombia, Chile, Guatemala, Perú y El Salvador; entre un 50-75\% en Brasil, Costa Rica, Cuba, Ecuador, Panamá, Puerto Rico, República Dominicana y Uruguay; y menos del 50\% en Argentina, México y 
TASAS DE ACTIVIDAD DE LA EMIGRACIÓN ESPAÑOLA A LATINOAMÉRICA

SEGÚN PAÍS DE DESTINO (1968-1988)

\begin{tabular}{|lc|}
\hline PAÍS & TASA ACTIVIDAD \\
\hline ARGENTINA & 52,5 \\
BOLIVIA & 93,6 \\
BRASIL & 62,0 \\
COLOMBIA & 74,5 \\
COSTA RICA & 55,4 \\
CUBA & 91,2 \\
CHILE & 79,6 \\
ECUADOR & 72,1 \\
GUATEMALA & 88,0 \\
MÉXICO & 48,6 \\
PANAMÁ & 56,9 \\
PERÚ & 90,0 \\
PUERTO RICO & 69,3 \\
R. DOMINICANA & 72,2 \\
EL SALVADOR & 63,8 \\
URUGUAY & 57,5 \\
VENEZUELA & 39,2 \\
\hline
\end{tabular}

FuENTE: E.E.A., 1968-1980; D.E.E.E., 1980-1986 y 1989; M.A.I.E.E., 1987-1988; A.M., 1992. Elaboración propia.

Figura 4. Distribución por ramas de actividad de la emigración española asistida a Latinoamérica 1968-1988.

Venezuela. Mientras en el primer y segundo conjunto de países, los profesionales y técnicos son seguidos en importancia por los obreros industriales; en el tercero aparecen algunas diferencias, así en el caso de Argentina, los profesionales y técnicos son seguidos por los obreros industriales, pero en México son superados por los empleados de oficina y 


\section{CUADRO VII}

DISTRIBUCIÓN POR RAMAS DE ACTIVIDAD DE LA EMIGRACIÓN ESPAÑOLA A LATINOAMÉRICA SEGÚN PAÍS DESTINO (1968-1988) (Cifras absolutas)

\begin{tabular}{|c|c|c|c|c|c|c|c|c|c|c|}
\hline PAÍS & 1 & 2 & 3 & 4 & 5 & 6 & 7 & 8 & 9 & X \\
\hline ARGENTINA & 1.197 & 14 & 276 & 374 & 395 & 13 & 70 & 827 & 53 & 291 \\
\hline BOLIVIA & 856 & 0 & 5 & 3 & 1 & 2 & 0 & 13 & 1 & 8 \\
\hline BRASIL & 1.436 & 5 & 86 & 283 & 67 & 12 & 29 & 532 & 38 & 170 \\
\hline COLOMBIA & 1.327 & 3 & 69 & 36 & 9 & 3 & 6 & 225 & 24 & 59 \\
\hline COSTA RICA & 161 & 1 & 13 & 1 & 2 & 0 & 0 & 2 & 36 & 11 \\
\hline CUBA & 50 & 0 & 2 & 0 & 0 & 0 & 3 & 33 & 2 & 3 \\
\hline CHILE & 936 & 3 & 24 & 22 & 23 & 0 & 4 & 63 & 7 & 26 \\
\hline ECUADOR & 954 & 4 & 44 & 21 & 2 & 8 & 1 & 186 & 15 & 60 \\
\hline GUATEMALA & 484 & 0 & 13 & 3 & 2 & 1 & 0 & 90 & 4 & 6 \\
\hline MÉXICO & 415 & 52 & 542 & 519 & 268 & 17 & 41 & 331 & 36 & 183 \\
\hline PANAMÁ & 228 & 2 & 33 & 35 & 31 & 0 & 6 & 66 & 3 & 30 \\
\hline PERÚ & 2.235 & 4 & 38 & 52 & 2 & 1 & 0 & 90 & 12 & 40 \\
\hline PUERTO RICO & 117 & 0 & 11 & 11 & 0 & 0 & 3 & 40 & 7 & 10 \\
\hline R. DOMINICANA & 531 & 2 & 19 & 11 & 13 & 6 & 2 & 115 & 6 & 17 \\
\hline SALVADOR & 116 & 0 & 3 & 1 & 1 & 0 & 0 & 21 & 5 & 1 \\
\hline URUGUAY & 263 & 1 & 31 & 40 & 61 & 4 & 9 & 82 & 7 & 19 \\
\hline VENEZUELA & 1.505 & 6 & 812 & 324 & 2.487 & 109 & 277 & 3.143 & 260 & 1.413 \\
\hline TOTAL & 12.811 & 97 & 2.021 & 1.736 & 3.364 & 176 & 451 & 5.859 & 516 & 2.347 \\
\hline ARGENTINA & 34,1 & 0,4 & 7,9 & 10,7 & 11,3 & 0,4 & 2,0 & 23,6 & 1,5 & 8,3 \\
\hline BOLIVIA & 96,3 & 0,0 & 0,6 & 0,3 & 0,1 & 0,2 & 0,0 & 1,5 & 0,1 & 0,9 \\
\hline BRASIL & 54,0 & 0,2 & 3,2 & 10,6 & 2,5 & 0,5 & 1,1 & 20,0 & 1,4 & 6,4 \\
\hline COLOMBIA & 75,4 & 0,2 & 3,9 & 2,0 & 0,5 & 0,2 & 0,3 & 12,8 & 1,4 & 3,4 \\
\hline COSTA RICA & 70,9 & 0,4 & 5,7 & 0,4 & 0,9 & 0,0 & 0,0 & 0,9 & 15,9 & 4,8 \\
\hline CUBA & 53,8 & 0,0 & 2,2 & 0,0 & 0,0 & 0,0 & 3,2 & 35,5 & 2,2 & 3,2 \\
\hline CHILE & 84,5 & 0,3 & 2,2 & 2,0 & 2,1 & 0,0 & 0,4 & 5,7 & 0,6 & 2,3 \\
\hline ECUADOR & 73,7 & 0,3 & 3,4 & 1,6 & 0,2 & 0,6 & 0,1 & 14,4 & 1,2 & 4,6 \\
\hline GUATEMALA & 80,3 & 0,0 & 2,2 & 0,5 & 0,3 & 0,2 & 0,0 & 14,9 & 0,7 & 1,0 \\
\hline MÉXICO & 17,3 & 2,2 & 22,5 & 21,6 & 11,1 & 0,7 & 1,7 & 13,8 & 1,5 & 7,6 \\
\hline PANAMÁ & 52,5 & 0,5 & 7,6 & 8,1 & 7,1 & 0,0 & 1,4 & 15,2 & 0,7 & 6,9 \\
\hline PERÚ & 90,3 & 0,2 & 1,5 & 2,1 & 0,1 & 0,0 & 0,0 & 3,6 & 0,5 & 1,6 \\
\hline PUERTO RICO & 58,8 & 0,0 & 5,5 & 5,5 & 0,0 & 0,0 & 1,5 & 20,1 & 3,5 & 5,0 \\
\hline R. DOMINICANA & 73,5 & 0,3 & 2,6 & 1,5 & 1,8 & 0,8 & 0,3 & 15,9 & 0,8 & 2,4 \\
\hline SALVADOR & 78,4 & 0,0 & 2,0 & 0,7 & 0,7 & 0,0 & 0,0 & 14,2 & 3,4 & 0,7 \\
\hline URUGUAY & 50,9 & 0,2 & 6,0 & 7,7 & 11,8 & 0,8 & 1,7 & 15,9 & 1,4 & 3,7 \\
\hline VENEZUELA & 14,6 & 0,1 & 7,9 & 3,1 & 24,1 & 1,1 & 2,7 & 30,4 & 2,5 & 13,7 \\
\hline TOTAL & 43,6 & 0,3 & 6,9 & 5,9 & 11,5 & 0,6 & 1,5 & 19,9 & 1,8 & 8,0 \\
\hline
\end{tabular}

1. Profesionales y Técnicos; 2. Administradores, gerentes y directores; 3. Empleados de Oficina; 4. Vendedores; 5. Agricultores, pescadores, cazadores, y silvicultores; 6. Minas y Canteras; 7. Transporte y comunicaciones; 8. Artesanos y obreros industriales; 9. Otros Servicios, deportes y diversiones; X. No clasificables;

FuENTE: E.E.A., 1968-1980; D.E.E.E., 1980-1986 y 1989; M.A.I.E.E., 1987-1988; A.M., 1992. Elaboración propia. 
FIGURA 5. Distribución regional de la emigración española asistida a Latinoamérica, 1965-1990.

Cuadro VIII

DISTRIBUCIÓN REGIONAL DE LA EMIGRACIÓN ESPAÑOLA A LATINOAMÉRICA (1965-1990)

\begin{tabular}{|lrrrrrr|}
\hline REGIONES & $\mathbf{1 9 6 5 - 7 0}$ & $\mathbf{\%}$ & $\mathbf{1 9 7 1 - 9 0}$ & $\boldsymbol{\%}$ & TOTAL & \% \\
\hline ANDALUCÍA & 1.305 & 2,9 & 1.305 & 4,0 & 2.610 & 3,3 \\
ARAGÓN & 568 & 1,3 & 475 & 1,4 & 1.043 & 1,3 \\
ASTURIAS & 1.120 & 2,5 & 533 & 1,6 & 1.653 & 2,1 \\
BALEARES & 234 & 0,5 & 164 & 0,5 & 398 & 0,5 \\
CANARIAS & 12.452 & 27,9 & 8.030 & 24,4 & 20.482 & 26,4 \\
CANTABRIA & 406 & 0,9 & 151 & 0,5 & 557 & 0,7 \\
CASTILLA-LA MANCHA & 177 & 0,4 & 211 & 0,6 & 388 & 0,5 \\
CASTILLA-LEÓN & 1.163 & 2,6 & 841 & 2,6 & 2.004 & 2,5 \\
CATALUÑA & 3.721 & 8,3 & 1.928 & 5,9 & 5.649 & 7,2 \\
EXTREMADURA & 63 & 0,1 & 107 & 0,3 & 170 & 0,2 \\
GALICIA & 16.935 & 38,0 & 7.513 & 22,8 & 24.448 & 31,5 \\
MADRID & 4.829 & 10,8 & 10.352 & 31,5 & 15.181 & 19,5 \\
MURCIA & 102 & 0,2 & 85 & 0,3 & 187 & 0,2 \\
NAVARRA & 257 & 0,6 & 246 & 0,7 & 503 & 0,6 \\
PAÍS VASCO & 247 & 0,6 & 353 & 1,1 & 600 & 0,7 \\
RIOJA & 138 & 0,3 & 69 & 0,2 & 207 & 0,2 \\
VALENCIA & 895 & 2,0 & 550 & 1,7 & 1.445 & 1,8 \\
ESPAÑA & 44.612 & 100,0 & 32.913 & 100,0 & 77.525 & 100,0 \\
\hline
\end{tabular}

FuENTE: A.M., 1992. Elaboración propia.

vendedores, al igual que en Venezuela donde presentan mayor proporción los obreros industriales y trabajadores del sector primario ${ }^{12}$.

12 La importancia del sector primario en la emigración española a Venezuela debemos ponerla en relación con la emigración canaria que recibe este país y que procede habitualmente de zonas agrícolas. 


\section{Procedencia regional de la emigración española asistida, 1965-1990}

Tres son las regiones que más emigrantes aportan en este período al movimiento emigratorio español asistido a Latinoamérica: las tradicionales Galicia (31\%) y Canarias (26\%), y Madrid (20\%), que son, a su vez, las únicas regiones que superan la cifra de diez mil emigrantes al final del período. El resto de regiones aporta cantidades decrecientes de emigrantes hasta el punto que ocho de ellas no superan el millar (País Vasco, Cantabria, Navarra, Baleares, Castilla-La Mancha, La Rioja, Murcia y Extremadura) (Cuadro VIII).

Ahora bien, si antes de 1970 la mayoría de los emigrantes proceden de Galicia (38\%) y Canarias $(27 \%)$, la reducción y especialización cada vez mayor de la emigración española asistida a partir de 1971 determina que Madrid pase a ocupar el primer lugar, siendo la única región que aporta más de diez mil emigrantes; pasando Galicia y Canarias a ocupar un segundo puesto, seguidas de Andalucía y Cataluña, únicas regiones que también superan el millar de emigrantes en la etapa 1971-90 (Cuadro VIII).

\section{Bibliografía}

GOZÁLVEZ PÉREZ, V., 1990: «El reciente incremento de la población extranjera en España y su incidencia laboral». INVESTIGACIONES GEOGRÁFICAS, 8, 7-36.

INSTITUTO ESPAÑOL DE EMIGRACIÓN (I.E.E.), 1985: Relación de convenios internacionales suscritos por España con América del Norte, América del Sur y Centroamérica. Madrid. Inédito.

PALAZÓN FERRANDO, S., 1989a: «Dificultades para el conocimiento real de la emigración española a Latinoamérica, 1970-1986», en VV.AA., Análisis del desarrollo de la población española 1970-1986, Barcelona, Síntesis, pp. 177-184.

PALAZÓN FERRANDO, S., 1989b: «La población española en América Latina. El resultado de una emigración». ESTUDIOS GEOGRÁFICOS, 197, pp. 607-707.

TORREALBA, R. y OROPEZA, J. A., 1988: Estado y migraciones laborales en Venezuela. Caracas. Ed. Cabildo, 192 pp.

VÁZQUEZ MATEO, F., 1968: La nueva emigración a Iberoamérica. Madrid. I.E.E., 101 pp. 